Jianying Miao, Ka Wai Wong, Wei Li, Shing Hang Ng, Lok Hang Keung, King Ho So, Iris Y.M. Ho, Ricky K.C. Luk, Lifeng Cai, Candy Cheng, Gena Y.Y. Tsang and Peter W. Lee

\title{
Nanomaterials applications in "green" functional coatings
}

ABSTRACT. The global coating market is huge, worth over US\$100 billion annually, with applications for physical and chemical protection, decoration and various other functions. In the last decade, the trend is definitely pointing toward the replacement of traditional VOC (volatile organic chemical)-based paints and polluting processes like electroplating with environmentally friendly materials and technologies. Nanomaterials play a significant role in the new generation of "green" functional coatings by providing specific functionalities to the base coating. For the replacement of electroplated metal coatings, a multilayer coating stack providing anticorrosion, mirror-like reflective and antiscratch functions was developed. Nanosized metal and ceramic particles are used to achieve these functions without the use of any polluting chemicals nor the release of any heavy metal contamination typical from electroplating processes. Furthermore, a multifunctional environmental paint was developed for wood surfaces. The key ingredient in this water-based paint is mesoporous silica nanoparticles, which offers high water resistance and a short drying time. This versatile material also offers high chemical tunability, which allows the incorporation of various additives to achieve multiple functions including antibacterial action and resistance to fire, household chemicals and UV (ultraviolet) exposure.

Nanotechnology Perceptions 8 (2012) 181-189

Nonsubscribers: purchase individual article 